\title{
THE ALGEBRAIC FUNCTIONAL EQUATION OF AN ELLIPTIC CURVE AT SUPERSINGULAR PRIMES
}

\author{
ByOung Du (B.D.) KIM
}

\begin{abstract}
Since the analytic functional equation holds for the \pm - $p$-adic $L$-functions constructed in [7], the algebraic functional equation for the \pm -Selmer groups is expected to hold as well. In this paper, we show it following the ideas of [1] and [4].
\end{abstract}

\section{Introduction}

We let $E$ be an elliptic curve defined over $\mathbb{Q}$ and let $p>3$ be a prime at which $E$ has good supersingular reduction. We let $K$ be an abelian extension of $\mathbb{Q}$ such that $[K: \mathbb{Q}]$ is prime to $p$ and $p$ is unramified over $K / \mathbb{Q}$.

Let $K_{\infty}$ be the cyclotomic $\mathbb{Z}_{p}$-extension of $K$. We define $S e l_{p}^{-}\left(E / K_{\infty}\right)$ following [5], [2], and [4]. We will explain this construction in the following sections.

Throughout this paper we use the following notation: Let $g=[K: \mathbb{Q}], O=\mathbb{Z}_{p}\left[\mu_{g}\right]$, and $F=\mathbb{Q}_{p}\left(\mu_{q}\right)$. Let $\Gamma=\operatorname{Gal}\left(K_{\infty} / K\right), \Lambda=\mathbb{Z}_{p}[[\Gamma]]$, and $\Lambda_{O}=\Lambda \otimes O$ (the reason for tensoring with $O$ will be explained later in this introduction). We identify $\Lambda_{O}$ with the integral power series ring $O[[X]]$ by identifying a topological generator $\gamma$ of $\Gamma$ with $1+X$. When $M$ is a $O$-module, we let $M^{\vee}$ denote the $O$-Pontryagin dual $\operatorname{Hom}_{O}(M, F / O)$ where $\operatorname{Hom}_{O}$ is the set of continuous $O$-homomorphisms.

Using Kato's and Rohrlich's work we will show $\operatorname{Sel}_{p}^{-}\left(E / K_{\infty}\right)$ is $\Lambda$-cotorsion, and following Greenberg's idea we will show

$$
\left(\operatorname{Sel}_{p}^{-}\left(E / K_{\infty}\right) \otimes O\right)^{\vee} \sim\left(\operatorname{Sel}_{p}^{-}\left(E / K_{\infty}\right)^{\iota} \otimes O\right)^{\vee}
$$

where $\sim$ is a $O\left[\left[\operatorname{Gal}\left(K_{\infty} / \mathbb{Q}\right)\right]\right]$-pseudo-isomorphism (a homomorphism with finite kernel and cokernel) and $\iota$ is the standard involution given by $g \rightarrow g^{-1}$ for any $g \in \operatorname{Gal}\left(K_{\infty} / \mathbb{Q}\right)$.

This implies that the characteristic ideal $(a) \subset \Lambda$ of the Pontryagin dual of $\operatorname{Sel}_{p}^{-}\left(E / K_{\infty}\right)$ is nonzero and satisfies the algebraic functional equation $(a)=\left(a^{\iota}\right)$. Pollack showed the analytic counterpart of this result, the analytic functional equation of minus- $p$-adic $L$-functions. (See [7] Theorem 5.13. He also proved that of plus-p-adic $L$-functions.) The main conjecture of Iwasawa theory of \pm -Selmer groups (see [5]) implies the analytic functional equation is equivalent to the algebraic functional equation.

Furthermore it is possible to formulate and prove one divisibility of the main conjecture of Iwasawa theory for $\mathrm{Sel}_{p}^{-}\left(E / K_{\infty}\right)$ similar to [5], in which one divisibility of that conjecture for $\operatorname{Sel}_{p}^{ \pm}\left(E / \mathbb{Q}\left(\mu_{p^{\infty}}\right)\right)$ was proven. (The proof is very similar to [5], and we will omit it.)

Received by the editors September 28, 2006. Revision received March 27, 2007. 
Applying our technique to the plus Selmer groups might be a little difficult. The construction of plus norm subgroups in [4] does not seem to always work unlike minus norm subgroups. However, when $K=\mathbb{Q}\left(\mu_{p}\right)$ or $p$ splits completely over $K / \mathbb{Q}$, we have the plus norm subgroups as constructed in [5] and [2], and we can prove the algebraic functional equation for the plus Selmer groups without modifying our technique.

This paper uses the idea of [1] which we now recall.

We let $\Delta=\operatorname{Gal}(K / \mathbb{Q})$ and $\mathbb{Q}_{\infty}=K_{\infty}^{\Delta}$. We let $\mathbb{Q}_{n}$ denote the subfield of $\mathbb{Q}_{\infty}$ with $\operatorname{Gal}\left(\mathbb{Q}_{n} / \mathbb{Q}\right) \cong \mathbb{Z} / p^{n} \mathbb{Z}$ (similarly $K_{n}$ denotes the subfield of $K_{\infty}$ with $\operatorname{Gal}\left(K_{n} / K\right) \cong$ $\left.\mathbb{Z} / p^{n} \mathbb{Z}\right)$, and $\Gamma_{n}$ denote $\operatorname{Gal}\left(K_{\infty} / K_{n}\right)$.

We let $A$ denote $E\left[p^{\infty}\right] \otimes O$. For a character $\eta$ of $\Delta$ we let $\epsilon_{\eta}=\sum_{\sigma \in \Delta} \eta\left(\sigma^{-1}\right) \sigma$ and $A_{\eta}$ be $A$ with action twisted by $\eta$. (As you might have noticed, to twist the action of $\Delta$ by $\eta$, we need to tensor $E\left[p^{\infty}\right]$ with $O$.)

The group $\Delta$ acts naturally on $\operatorname{Sel}_{p}^{-}\left(E / K_{\infty}\right)$. Since $[K: \mathbb{Q}]$ is prime to $p$, we have the decomposition $\operatorname{Sel}_{p}^{-}\left(E / K_{\infty}\right) \otimes O \cong \oplus_{\eta} \epsilon_{\eta}\left(\operatorname{Sel}_{p}^{-}\left(E / K_{\infty}\right) \otimes O\right)$ where $\eta$ runs over all characters of $\Delta$. Thus to show

$$
\operatorname{Sel}_{p}^{-}\left(E / K_{\infty}\right) \otimes O \sim \operatorname{Sel}_{p}^{-}\left(E / K_{\infty}\right)^{\iota} \otimes O
$$

as $O\left[\left[\operatorname{Gal}\left(K_{\infty} / \mathbb{Q}\right)\right]\right]$-modules, it is enough to show

$$
\epsilon_{\eta}\left(\operatorname{Sel}_{p}^{-}\left(E / K_{\infty}\right) \otimes O\right) \sim \epsilon_{\bar{\eta}}\left(S e l_{p}^{-}\left(E / K_{\infty}\right)^{\iota} \otimes O\right)
$$

as $\Lambda_{O}$-modules for each character $\eta$ of $\Delta$.

To do so, we will define a local condition $H_{\mathcal{F}}^{1}\left(\mathbb{Q}_{\infty, v}, A_{\eta}\right)$ for every place $v$ of $\mathbb{Q}_{\infty}$ such that the group $S_{\eta}=H_{\mathcal{F}}^{1}\left(\mathbb{Q}_{\infty}, A_{\eta}\right)$ associated to this local condition is isomorphic to $\left(\operatorname{Sel}_{\bar{p}}^{-}\left(E / K_{\infty}\right) \otimes O\right)^{\bar{\eta}}$. By Iwasawa theory (see proposition 3.6), to show $S_{\eta} \sim S_{\bar{\eta}}^{\iota}$, it is sufficient to show that $\operatorname{corank}_{O}\left(S_{\eta} \otimes \Lambda_{O} /(f)\right)^{\Gamma}=\operatorname{corank}_{O}\left(S_{\bar{\eta}} \otimes \Lambda_{O} /\left(f^{\iota}\right)\right)^{\Gamma}$ for every monic polynomial $f \in \Lambda_{O}$ and that $\left|S_{\eta}^{\Gamma_{m}}\left[p^{n}\right]\right| /\left|S_{\bar{\eta}}^{\Gamma_{m}}\left[p^{n}\right]\right|$ is bounded as $m$ and $n$ vary. The critical part in establishing this is to show the local conditions satisfy duality with respect to the local pairings (proposition 2.5).

Remark 1.1. For an $O$-module $M$ with $\Delta$-action and a character $\eta$ of $\Delta$, we let $M^{\eta}$ denote the submodule of $M$ where every $\sigma \in \Delta$ acts as multiplication by $\eta(\sigma)$. In fact, we can identify $M^{\eta}=\epsilon_{\eta} M$ and will use them interchangeably.

\section{The minus decomposition of a formal group}

As we mentioned earlier, let $K_{\infty}$ be the cyclotomic $\mathbb{Z}_{p^{-}}$extension of $K$. In other words $K_{\infty}$ is $K\left(\mu_{p^{\infty}}\right)^{\Delta^{\prime}}$ where $\operatorname{Gal}\left(K\left(\mu_{p^{\infty}}\right) / K\right) \cong \Gamma \times \Delta^{\prime}$ with $\Gamma \cong \mathbb{Z}_{p}$ and a torsion subgroup $\Delta^{\prime}$. We let $K_{n}$ denote the subfield of $K_{\infty}$ with $\operatorname{Gal}\left(K_{n} / K\right) \cong \mathbb{Z} / p^{n} \mathbb{Z}$.

Suppose $P$ is a prime of $K$ lying above $p$. Let $k$ be $K_{P}, k_{n}$ be $K_{n, q}$ where $q$ denotes the unique prime of $K_{n}$ lying above $P$, and $k_{\infty}$ be $\cup_{n=0}^{\infty} k_{n}$ (also let $k_{-1}=k$ ).

For an extension $L$ of $\mathbb{Q}_{p}$ we let $O_{L}$ denote the ring of integers of $L$, and $m_{L}$ denote the unique maximal ideal of $O_{L}$. Let $\hat{E}$ be the formal group over $\mathbb{Z}_{p}$ associated to $E$. We let $\hat{E}(L)$ denote $\hat{E}\left(m_{L}\right)$.

Definition 2.1. We define 


$$
\begin{gathered}
\hat{E}^{-}\left(k_{n}\right):=\left\{x \in \hat{E}\left(k_{n}\right) \mid T r_{n / m+1} x \in \hat{E}\left(k_{m}\right) \text { for all }-1 \leq m<n, m \text { odd }\right\}, \\
\mathbb{H}=\cup_{n=0}^{\infty} \hat{E}^{-}\left(k_{n}\right) \otimes \mathbb{Q}_{p} / \mathbb{Z}_{p}, \\
\mathbb{H}_{n}=\mathbb{H}^{\Gamma_{n}} .
\end{gathered}
$$

From [4] we have the following.

Proposition 2.2. $\quad$ (1) Let $\Lambda_{P}$ denote $\mathbb{Z}_{p}\left[\left[\operatorname{Gal}\left(k_{\infty} / k\right)\right]\right]$. We have

$$
\operatorname{Hom}\left(\mathbb{H}, \mathbb{Q}_{p} / \mathbb{Z}_{p}\right) \cong \Lambda_{P}^{\left[K_{P}: \mathbb{Q}_{p}\right]} \text {. }
$$

(2) For any integer $m, \mathbb{H}_{n}\left[p^{m}\right]$ is the exact annihilator of itself with respect to the Tate local pairing

$$
H^{1}\left(k_{n}, E\left[p^{m}\right]\right) \times H^{1}\left(k_{n}, E\left[p^{m}\right]\right) \rightarrow \mathbb{Z} / p^{m} \mathbb{Z} .
$$

Proof. This is precisely [4] propositions 3.13 and 3.15 since $k_{\infty} / k$ is a totally ramified extension. You can also see [4] propositions 3.17 and 3.18 .

Let $f(X)$ be a monic distinguished polynomial of $\Lambda_{O}$ (i.e. $f(X)=X^{k}+a_{1} X^{k-1}+$ $\cdots+a_{k}$ where $p \mid a_{i}$ for every $\left.i\right)$.

Let $Y_{f}$ denote $\Lambda_{O} /(f(X))$ and $\Lambda_{O}$ act on $\operatorname{Hom}_{O}\left(Y_{f}, O\right)$ as follows: for $\sigma \in \Gamma$ and $\phi \in \operatorname{Hom}_{O}\left(Y_{f}, O\right),(\sigma \circ \phi)(x)=\phi\left(\sigma^{-1} x\right)$. Then $\operatorname{Hom}_{O}\left(Y_{f}, O\right)$ is isomorphic to $Y_{f^{\iota}}=\Lambda_{O} / f^{\iota}(X)$ as a $\Lambda_{O}$-module.

We recall $A=E\left[p^{\infty}\right] \otimes O$ and for a character $\eta$ of $\Delta, A_{\eta}$ is $A$ with action twisted by $\eta$. We let $A_{f}$ denote $A \otimes_{O} Y_{f}$ and $A_{f, \eta}$ denote $A_{\eta} \otimes_{O} Y_{f}$. The following is essentially from [5] proposition 8.7.

Lemma 2.3. We have $A^{G_{k_{\infty}}}=0$.

Proof. Let $F$ be the unramified quadratic extension of $\mathbb{Q}_{p}$ and $x$ be any nontrivial $p$-torsion of $\hat{E}$. [5] proposition 8.6 shows $\hat{E}$ is isomorphic over $O_{F}$ to a Lubin-Tate group of height 2 , thus $F(x)$ is a totally ramified extension of $F$ of degree $p^{2}-1$. Since we assume $k$ is an unramified extension of $\mathbb{Q}_{p}, k F$ is also unramified over $\mathbb{Q}_{p}$. Therefore $\hat{E}(k F)$ does not contain $x$. In other words, $\hat{E}(k F)[p]=0$.

On the other hand, $\hat{E}\left(k_{n}\right)[p]$ can be written as a union of disjoint orbits $\cup_{i}\left[x_{i}\right]$ where $\left[x_{i}\right]$ denotes an orbit $\left\{x_{i}^{\sigma} \mid \sigma \in \operatorname{Gal}\left(k_{n} / k\right)\right\}$. If $\operatorname{Gal}\left(k_{n} / k\right)$ does not act trivially on $x_{i}$, the order of $\left[x_{i}\right]$ is divisible by $p$. Since $\hat{E}(k)[p]=0$, the only point on which $\operatorname{Gal}\left(k_{n} / k\right)$ acts trivially is 0 . Therefore the order of $\hat{E}\left(k_{n}\right)[p]$ is not divisible by $p$. Hence $\hat{E}\left(k_{n}\right)[p]=0$.

Since $E$ has good supersingular reduction at $p$, we have $E[p]=\hat{E}[p]$; therefore we have $E\left(k_{n}\right)[p]=0$. Since $G_{k_{\infty}}$ acts trivially on $O$ of $A=E\left[p^{\infty}\right] \otimes O$, we have $A^{G_{k_{\infty}}}=0$. 
Since $G_{k_{\infty}}$ acts trivially on $Y_{f}$, we have $A_{f}^{G_{k_{\infty}}}=0$ and $A_{f, \eta}^{G_{k}}=0$. Thus from the Serre-Hochschild spectral sequence we have

$$
H^{1}\left(k_{n}, A_{f}\right) \stackrel{\sim}{\rightarrow} H^{1}\left(k_{\infty}, A_{f}\right)^{\Gamma_{n}}
$$

For any integer $m$, we have a short exact sequence

$$
0 \rightarrow A_{f}\left[p^{m}\right] \rightarrow A_{f} \stackrel{p^{m}}{\rightarrow} A_{f} \rightarrow 0 .
$$

This sequence induces a long exact sequence of cohomology groups. Combined with $A_{f}^{G_{k_{n}}}=0$, this long exact sequence induces

$$
H^{1}\left(k_{n}, A_{f}\left[p^{m}\right]\right) \stackrel{\sim}{\rightarrow} H^{1}\left(k_{n}, A_{f}\right)\left[p^{m}\right] .
$$

We identify $H^{1}\left(k_{n}, A_{f}\right)$ with $H^{1}\left(k_{\infty}, A_{f}\right)^{\Gamma_{n}}$ and $H^{1}\left(k_{n}, A_{f}\left[p^{m}\right]\right)$ with $H^{1}\left(k_{n}, A_{f}\right)\left[p^{m}\right]$. We define the following.

Definition 2.4. We define

$$
\begin{gathered}
\mathbb{H}_{f}:=\mathbb{H} \otimes Y_{f} \subset H^{1}\left(k_{\infty}, E\left[p^{\infty}\right]\right) \otimes Y_{f}=H^{1}\left(k_{\infty}, A_{f}\right), \\
\mathbb{H}_{f}^{n}\left[p^{m}\right]:=\mathbb{H}_{f}\left[p^{m}\right]^{\Gamma_{n}} \subset H^{1}\left(k_{n}, A_{f}\left[p^{m}\right]\right) .
\end{gathered}
$$

Since $Y_{f^{\iota}}$ is isomorphic to $\operatorname{Hom}_{O}\left(Y_{f}, O\right)$, there is a natural pairing $Y_{f} \times Y_{f^{\iota}} \rightarrow O$. When we let $G_{K}$ act on $Y_{f}$ through the canonical map $G_{K} \rightarrow \Gamma \rightarrow \Lambda$ and act on $O$ trivially, we can check that this pairing is an $O$-linear $G_{K}$-equivariant perfect pairing. Combined with the Weil pairing $E\left[p^{m}\right] \times E\left[p^{m}\right] \rightarrow \mathbb{Z} / p^{m} \mathbb{Z}(1)$, we have an $O$-linear $G_{K^{-}}$equivariant perfect pairing $A_{f}\left[p^{m}\right] \times A_{f^{\iota}}\left[p^{m}\right] \rightarrow O / p^{m} O(1)$. By the cup product this induces a local pairing

$$
(,)_{n}: H^{1}\left(k_{n}, A_{f}\left[p^{m}\right]\right) \times H^{1}\left(k_{n}, A_{f^{\iota}}\left[p^{m}\right]\right) \rightarrow H^{2}\left(k_{n}, O / p^{m} O(1)\right) \stackrel{i n v}{\longrightarrow} O / p^{m} O .
$$

We will prove the following proposition.

Proposition 2.5. For any integer $n \geq 0, \mathbb{H}_{f}^{n}\left[p^{m}\right]$ is the exact annihilator of $\mathbb{H}_{f^{\iota}}^{n}\left[p^{m}\right]$ with respect to the pairing above.

Proof. We let $M_{n}$ be the exact annihilator of $\mathbb{H}_{f}^{n}\left[p^{m}\right]$ with respect to $(,)_{n}$ for every integer $n \geq 0$. We consider the maps $\operatorname{Res}_{n}^{n+1}: H^{1}\left(k_{n}, A_{f}\left[p^{m}\right]\right) \rightarrow H^{1}\left(k_{n+1}, A_{f}\left[p^{m}\right]\right)$ and $\operatorname{Cor}_{n}^{n+1}: H^{1}\left(k_{n+1}, A_{f}\left[p^{m}\right]\right) \rightarrow H^{1}\left(k_{n}, A_{f}\left[p^{m}\right]\right)$. Similar to the discussion before definition 2.4 we can identify $H^{1}\left(k_{n}, A_{f}\left[p^{m}\right]\right)$ with its image under $\operatorname{Res}_{n}^{n+1}$ because $\operatorname{Res}_{n}^{n+1}$ is injective. From [4] proposition 2.1 we have $\operatorname{Res}_{n}^{n+1} \circ \operatorname{Cor}_{n}^{n+1}=N_{n+1 / n}$. Thus we have

$$
\operatorname{Res}_{n}^{n+1} \circ \operatorname{Cor}_{n}^{n+1}\left(\mathbb{H}_{f}^{n+1}\left[p^{m}\right]\right) \subset \mathbb{H}_{f}^{n+1}\left[p^{m}\right]^{\operatorname{Gal}\left(k_{n+1} / k_{n}\right)}=\mathbb{H}_{f}^{n}\left[p^{m}\right] .
$$

Inductively we have $\operatorname{Cor}_{n}^{n^{\prime}} \mathbb{H}_{f}^{n^{\prime}}\left[p^{m}\right] \subset \mathbb{H}_{f}^{n}\left[p^{m}\right]$ for any integer $n^{\prime}>n$.

Let $j>n$ be an integer large enough so that $G_{k_{j}}$ acts trivially on $Y_{f} / p^{m} Y_{f}$. Combined with proposition 2.2.(2), it implies $M_{j}=\mathbb{H}_{f^{\iota}}^{j}\left[p^{m}\right]$. For $i \leq j$, by the 
property of cup product we have $\left(\operatorname{Cor}_{i}^{j} x, y\right)_{i}=\left(x, \operatorname{Res}_{i}^{j} y\right)_{j}$ for any $x \in H^{1}\left(k_{j}, A_{f}\left[p^{m}\right]\right)$ and $y \in H^{1}\left(k_{i}, A_{f^{\iota}}\left[p^{m}\right]\right)$. Assume $\left(\mathbb{H}_{f}^{n}\left[p^{m}\right], y\right)_{n}=0$ (equivalently $\left.y \in M_{n}\right)$. Then we have $\left(\mathbb{H}_{f}^{j}\left[p^{m}\right], \operatorname{Res}_{n}^{j} y\right)_{j}=0$ because $\operatorname{Cor}_{n}^{j} \mathbb{H}_{f}^{j}\left[p^{m}\right] \subset \mathbb{H}_{f}^{n}\left[p^{m}\right]$. Thus $\operatorname{Res}_{n}^{j} y \in M_{j}$, i.e., $M_{n} \subset M_{j}$ when we consider $H^{1}\left(k_{n}, A_{f^{\iota}}\left[p^{m}\right]\right)$ as a subgroup of $H^{1}\left(k_{j}, A_{f^{\iota}}\left[p^{m}\right]\right)$. More precisely we have $M_{n} \subset M_{j}^{\operatorname{Gal}\left(k_{j} / k_{n}\right)}$. Since we have $M_{j}=\mathbb{H}_{f^{\iota}}^{j}\left[p^{m}\right]$, we have $M_{n} \subset \mathbb{H}_{f^{\iota}}^{n}\left[p^{m}\right]$.

We can check

$$
\begin{aligned}
\left|M_{n}\right| & =\left|H^{1}\left(k_{n}, A_{f}\left[p^{m}\right]\right)\right| /\left|\mathbb{H}_{f}^{n}\left[p^{m}\right]\right| \\
& =\left|\mathbb{H}_{f^{\iota}}^{n}\left[p^{m}\right]\right| .
\end{aligned}
$$

Thus we have $M_{n}=\mathbb{H}_{f^{\iota}}^{n}\left[p^{m}\right]$.

\section{The algebraic functional equation}

We fix a finite set $\Sigma$ of places of $\mathbb{Q}$ which includes $p$, all primes of bad reduction of $E$, all primes ramified over $K / \mathbb{Q}$, and infinite places. For a number field $L$ and a set $\Omega$ of places of $\mathbb{Q}$ we let $L_{\Omega}$ denote the maximal extension of $L$ unramified outside the primes lying above $\Omega$. For any prime $P$ of $K_{n}(n \leq \infty)$ lying above $p$, by the Serre-Hochschild sequence we have

$$
\begin{aligned}
H^{1}\left(K_{n, P} / \mathbb{Q}_{n, p}, A_{f, \eta}^{G_{K_{n, P}}}\right) & \rightarrow H^{1}\left(\mathbb{Q}_{n, p}, A_{f, \eta}\right) \\
& \rightarrow H^{1}\left(K_{n, P}, A_{f, \eta}\right)^{\operatorname{Gal}\left(K_{n, P} / \mathbb{Q}_{n, p}\right)} \rightarrow H^{2}\left(K_{n, P} / \mathbb{Q}_{n, p}, A_{f, \eta}^{G_{K_{n, P}}}\right) .
\end{aligned}
$$

In the previous section we saw $A_{f}^{G_{K_{\infty}, P}}=0$, thus the first and last groups are trivial. Thus we can deduce

$$
H^{1}\left(\mathbb{Q}_{n, p}, A_{f, \eta}\right) \stackrel{\sim}{\rightarrow}\left(\prod_{P \mid p} H^{1}\left(K_{n, P}, A_{f, \eta}\right)\right)^{\Delta}=\epsilon_{\bar{\eta}} \prod_{P \mid p} H^{1}\left(K_{n, P}, A_{f}\right) .
$$

Proposition 3.1. Let $\mathbb{H}_{P}$ denote the group $\mathbb{H}$ in definition 2.1 for each $P \mid p$. We have an isomorphism of $\Lambda_{O}$-modules

$$
\left(\epsilon_{\eta} \cdot \prod_{P \mid p} \mathbb{H}_{P} \otimes O\right)^{\vee} \cong \Lambda_{O}
$$

Proof. Since $\mathbb{H}_{P}^{\Gamma} \cong \hat{E}\left(K_{P}\right) \otimes \mathbb{Q}_{p} / \mathbb{Z}_{p} \cong K_{P} / O_{K_{P}}$, we have $\epsilon_{\eta} \prod_{P \mid p}\left(\mathbb{H}_{P} \otimes O\right)^{\Gamma} \cong$ $\epsilon_{\eta} \prod_{P \mid p} K_{P} / O_{K_{P}} \otimes O \cong F / O$. Therefore by Nakayama's lemma the $O$-Pontryagin dual of $\epsilon_{\eta} \cdot \prod_{P \mid p} \mathbb{H}_{P} \otimes O$ is a $\Lambda_{O}$-module generated by one element, and our claim follows. 
Definition 3.2. We let $\mathbb{H}_{P, f}$ denote $\mathbb{H}_{P} \otimes Y_{f}$. For every $m, n \leq \infty$ we let $H_{\mathcal{F}}^{1}\left(\mathbb{Q}_{n, p}, A_{f, \eta}\left[p^{m}\right]\right)$ be the inverse image of $\epsilon_{\bar{\eta}} \prod_{P \mid p} \mathbb{H}_{P, f}\left[p^{m}\right]^{\Gamma_{n}}$ under the isomorphism

$$
H^{1}\left(\mathbb{Q}_{n, p}, A_{f, \eta}\left[p^{m}\right]\right) \rightarrow \epsilon_{\bar{\eta}} \prod_{P \mid p} H^{1}\left(K_{n, P}, A_{f}\left[p^{m}\right]\right) .
$$

For a local field $L$ and a $G_{L}$-module $B$, we let $H_{u r}^{1}(L, B)$ denote $H^{1}\left(L^{u r} / L, B^{L^{u r}}\right)$ where $L^{u r}$ is the maximal unramified extension of $L$. For a prime $w$ not lying above $p$ we let $H_{\mathcal{F}}^{1}\left(\mathbb{Q}_{n, w}, A_{f, \eta}\left[p^{m}\right]\right)=H_{u r}^{1}\left(\mathbb{Q}_{n, w}, A_{f, \eta}\left[p^{m}\right]\right)$.

We define

$$
H_{\mathcal{F}}^{1}\left(\mathbb{Q}_{n}, A_{f, \eta}\left[p^{m}\right]\right)=\operatorname{ker}\left(H^{1}\left(\mathbb{Q}_{\Sigma} / \mathbb{Q}_{n}, A_{f, \eta}\left[p^{m}\right]\right) \rightarrow \prod \frac{H^{1}\left(\mathbb{Q}_{n, v}, A_{f, \eta}\left[p^{m}\right]\right)}{H_{\mathcal{F}}^{1}\left(\mathbb{Q}_{n, v}, A_{f, \eta}\left[p^{m}\right]\right)}\right)
$$

where $v$ runs over all the primes of $\mathbb{Q}_{n}$ lying above $\Sigma$.

When $f=(X)$, we let $H_{\mathcal{F}}^{1}\left(\mathbb{Q}_{n}, A_{\eta}\left[p^{m}\right]\right)$ denote $H_{\mathcal{F}}^{1}\left(\mathbb{Q}_{n}, A_{f, \eta}\left[p^{m}\right]\right)$. We note that when $w$ is not lying above $p$, we have $H_{u r}^{1}\left(\mathbb{Q}_{\infty, w}, A_{\eta}\right)=0$ because $\mathbb{Q}_{\infty, w} / \mathbb{Q}_{v}$ is a $\mathbb{Z}_{p}$-extension.

Since $A_{f, \eta}^{G_{K_{\infty}, P}}=0$ for any prime $P$ of $K$ with $P \mid p$, we have $H^{1}\left(\mathbb{Q}_{n, p}, A_{f, \eta}\left[p^{m}\right]\right)=$ $H^{1}\left(\mathbb{Q}_{n, p}, A_{f, \eta}\right)\left[p^{m}\right]$, and we can check that under this identification we have

$$
H_{\mathcal{F}}^{1}\left(\mathbb{Q}_{n, p}, A_{f, \eta}\left[p^{m}\right]\right)=H_{\mathcal{F}}^{1}\left(\mathbb{Q}_{n, p}, A_{f, \eta}\right)\left[p^{m}\right] .
$$

The following commutative diagram is given by the property of cup product.

$$
\begin{array}{cccccc}
H^{1}\left(\mathbb{Q}_{n, p}, A_{f, \eta}\left[p^{m}\right]\right) & \times & H^{1}\left(\mathbb{Q}_{n, p}, A_{f^{\iota}, \bar{\eta}}\left[p^{m}\right]\right) & \rightarrow & O / p^{m} O \\
\downarrow & & \begin{array}{c}
\uparrow \\
\text { Ces }
\end{array} & & & \downarrow \\
\epsilon_{\bar{\eta}} \prod_{P \mid p} H^{1}\left(K_{n, P}, A_{f}\left[p^{m}\right]\right) & \times & \epsilon_{\eta} \prod_{P \mid p} H^{1}\left(K_{n, P}, A_{f^{\iota}}\left[p^{m}\right]\right) & \rightarrow & O / p^{m} O .
\end{array}
$$

(Commutativity means we have $(\operatorname{Res} x, y)=(x$, Cor $y)$.) Here Res is an isomorphism as discussed before proposition 3.1. Since Cor $\circ$ Res is multiplication by $[K: \mathbb{Q}]$ and $[K: \mathbb{Q}]$ is prime to $p$, we have

$$
\operatorname{Cor}\left(\epsilon_{\eta} \prod_{P \mid p} \mathbb{H}_{P, f^{\iota}}\left[p^{m}\right]^{\Gamma_{n}}\right)=\operatorname{Cor} \circ \operatorname{Res}\left(H_{\mathcal{F}}^{1}\left(\mathbb{Q}_{n, p}, A_{f^{\iota}, \bar{\eta}}\left[p^{m}\right]\right)\right)=H_{\mathcal{F}}^{1}\left(\mathbb{Q}_{n, p}, A_{f^{\iota}, \bar{\eta}}\left[p^{m}\right]\right) .
$$

Thus $H_{\mathcal{F}}^{1}\left(\mathbb{Q}_{n, p}, A_{f, \eta}\left[p^{m}\right]\right)$ is the exact annihilator of $H_{\mathcal{F}}^{1}\left(\mathbb{Q}_{n, p}, A_{f^{\iota}, \bar{\eta}}\left[p^{m}\right]\right)$. From [1] chapter 8 we have the following.

Lemma 3.3. For $m, n<\infty$ we have $\left|H_{\mathcal{F}}^{1}\left(\mathbb{Q}_{n}, A_{f, \eta}\left[p^{m}\right]\right)\right|=\left|H_{\mathcal{F}}^{1}\left(\mathbb{Q}_{n}, A_{f^{\iota}, \bar{\eta}}\left[p^{m}\right]\right)\right|$.

Proof. For a totally real field $F$ and a finite $G_{F}$-module $M$ let $\chi_{F}(M)$ denote the Euler characteristic $\left|H^{0}(F, M)\right| \cdot\left|H^{2}(F, M)\right| /\left|H^{1}(F, M)\right|$. Assume that the order of $M$ is prime to 2. Then it is known that $\chi_{F}(M)=1 /\left|M^{-}\right|^{[F: \mathbb{Q}]}$ where $M^{-}$is the maximal subgroup of $M$ where the complex conjugation acts by multiplication by -1 .

Let $F=\mathbb{Q}_{n}, M=A_{f, \eta}\left[p^{m}\right]$, and $M^{*}=A_{f^{\iota}, \bar{\eta}}\left[p^{m}\right]$. We can easily check $M^{*}=$ $\operatorname{Hom}_{O}\left(M, O / p^{m} O(1)\right)$. 
Following Greenberg ([1]) we use the following notation: we let

$$
\begin{aligned}
S:=H_{\mathcal{F}}^{1}(F, M), & S^{*}:=H_{\mathcal{F}}^{1}\left(F, M^{*}\right), \\
P_{\Sigma}^{i}:=\prod H^{i}\left(F_{v}, M\right), & P_{\Sigma}^{i, *}:=\prod H^{i}\left(F_{v}, M^{*}\right),
\end{aligned}
$$

(every product in this proof runs over all places $v$ of $F$ lying above $\Sigma$ unless mentioned otherwise),

$$
\begin{gathered}
L_{v}:=H_{\mathcal{F}}^{1}\left(F_{v}, M\right), \quad L_{v}^{*}:=H_{\mathcal{F}}^{1}\left(F_{v}, M^{*}\right), \\
L:=\prod L_{v}, \quad L^{*}:=\prod L_{v}^{*}, \\
\lambda^{i}: H^{i}\left(F_{\Sigma} / F, M\right) \rightarrow P_{\Sigma}^{i}, \\
G^{i}:=\operatorname{im} \lambda^{i}, \quad K^{i}=\operatorname{ker} \lambda^{i},
\end{gathered}
$$

(and define $\lambda^{i, *}, G^{i, *}$, and $K^{i, *}$ similarly).

Then we have

$$
|S|=\left|K^{1}\right| \cdot\left|G^{1} \cap L\right|=\left|K^{1}\right| \cdot\left|G^{1}\right| \cdot|L| \cdot\left|G^{1} \cdot L\right|^{-1} .
$$

We have $\left|K^{1}\right| \cdot\left|G^{1}\right|=\left|H^{1}\left(F_{\Sigma} / F, M\right)\right|$. By global duality $G^{1}$ is the exact annihilator of $G^{1, *}$ with respect to the local pairing between $P_{\Sigma}^{1}$ and $P_{\Sigma}^{1, *}$ (for a statement of global duality or Poitou-Tate duality see [6] Theorem I.4.10 or [10] Theorem 3.1).

If $v \mid p, L_{v}$ is the exact annihilator of $L_{v}^{*}$ by our previous discussion. If $v \nmid p$, it follows from the definition that $L_{v}$ is the exact annihilator of $L_{v}^{*}$. Hence we have $\left|G^{1} \cdot L\right|=\left|P_{\Sigma}^{1}\right| /\left|G^{1, *} \cap L^{*}\right|$. Therefore we have

$$
|S|=\left|H^{1}\left(F_{\Sigma} / F, M\right)\right| \cdot|L| \cdot \frac{\left|G^{1, *} \cap L^{*}\right|}{\left|P_{\Sigma}^{1}\right|} .
$$

From the definition of the global Euler characteristic we have

$$
\begin{aligned}
\left|H^{1}\left(F_{\Sigma} / F, M\right)\right| & =\chi_{F}(M)^{-1}\left|H^{0}\left(F_{\Sigma} / F, M\right)\right| \cdot\left|H^{2}\left(F_{\Sigma} / F, M\right)\right| \\
& =\chi_{F}(M)^{-1}\left|H^{2}\left(F_{\Sigma} / F, M\right)\right| .
\end{aligned}
$$

By global duality we have $\left|K^{1, *}\right|=\left|K^{2}\right|$, and thus we have

$$
\left|G^{1, *} \cap L^{*}\right|=\left|S^{*}\right| /\left|K^{1, *}\right|=\left|S^{*}\right| /\left|K^{2}\right| \text {. }
$$

On the other hand, by global duality we have $\mid$ coker $\lambda^{2}|=| H^{0}\left(F_{\Sigma} / F, M^{*}\right) \mid$; thus we have

$$
\frac{\left|H^{2}\left(F_{\Sigma} / F, M\right)\right|}{\left|K^{2}\right|}=\left|G^{2}\right|=\frac{\left|P_{\Sigma}^{2}\right|}{\left|\operatorname{coker} \lambda^{2}\right|}=\frac{\left|P_{\Sigma}^{2}\right|}{\left|H^{0}\left(F_{\Sigma} / F, M^{*}\right)\right|}=\left|P_{\Sigma}^{2}\right| .
$$

Then we check

$$
\begin{aligned}
\frac{|L|}{\left|P_{\Sigma}^{1}\right|} & =\frac{\prod_{v \nmid p}\left|H^{1}\left(F_{v}^{u r} / F_{v}, M^{I_{v}}\right)\right|}{\prod_{v \nmid p}\left|H^{1}\left(F_{v}, M\right)\right|} \prod_{P \mid p} \frac{\left|L_{P}\right|}{\left|H^{1}\left(F_{P}, M\right)\right|} \\
& =\prod_{v \nmid p} \frac{\left|H^{0}\left(F_{v}, M\right)\right|}{\left|H^{1}\left(F_{v}, M\right)\right|} \cdot \frac{1}{\left.|M|\right|^{n}[K: \mathbb{Q}]} .
\end{aligned}
$$


Since we have $\chi_{F}(M)^{-1}=|M|^{p^{n}[K: \mathbb{Q}]}$ and $\left|H^{1}\left(F_{v}, M\right)\right|=\left|H^{0}\left(F_{v}, M\right)\right| \cdot\left|H^{2}\left(F_{v}, M\right)\right|$ when $v \nmid p$, we obtain $|S|=\left|S^{*}\right|$.

Lemma 3.4. The kernel and cokernel of

$$
H_{\mathcal{F}}^{1}\left(\mathbb{Q}_{n}, A_{f, \eta}\left[p^{m}\right]\right) \rightarrow H_{\mathcal{F}}^{1}\left(\mathbb{Q}_{n}, A_{f, \eta}\right)\left[p^{m}\right]
$$

are finite and bounded as $m, n$ vary.

Proof. We consider the following diagram.

$$
\begin{array}{rrrr}
0 \rightarrow H_{\mathcal{F}}^{1}\left(\mathbb{Q}_{n}, A_{f, \eta}\left[p^{m}\right]\right) & \rightarrow H^{1}\left(\mathbb{Q}_{\Sigma} / \mathbb{Q}_{n}, A_{f, \eta}\left[p^{m}\right]\right) & \rightarrow & \prod_{v} \frac{H^{1}\left(\mathbb{Q}_{n, v}, A_{f, \eta}\left[p^{m}\right]\right)}{H_{\mathcal{F}}^{1}\left(\mathbb{Q}_{n, v}, A_{f, \eta}\left[p^{m}\right]\right)} \\
\downarrow & \downarrow & \downarrow \\
0 \rightarrow H_{\mathcal{F}}^{1}\left(\mathbb{Q}_{n}, A_{f, \eta}\right)\left[p^{m}\right] & \rightarrow H^{1}\left(\mathbb{Q}_{\Sigma} / \mathbb{Q}_{n}, A_{f, \eta}\right)\left[p^{m}\right] \rightarrow & \prod_{v} \frac{H^{1}\left(\mathbb{Q}_{n, v}, A_{f, \eta}\right)}{H_{\mathcal{F}}^{1}\left(\mathbb{Q}_{n, v}, A_{f, \eta}\right)}
\end{array}
$$

(every product in this proof runs over all primes lying above $\Sigma$ unless mentioned otherwise). The center vertical map is naturally surjective, and its kernel is $A_{f, \eta}^{G_{\mathbb{Q}_{n}}} / p^{m} A_{f, \eta}^{G_{\mathbb{Q}_{n}}}$ $=0$.

We let $f_{v, m}$ denote the $\operatorname{map} \frac{H^{1}\left(\mathbb{Q}_{n, v}, A_{f, \eta}\left[p^{m}\right]\right)}{H_{\mathcal{F}}^{1}\left(\mathbb{Q}_{n, v}, A_{f, \eta}\left[p^{m}\right]\right)} \rightarrow \frac{H^{1}\left(\mathbb{Q}_{n, v}, A_{f, \eta}\right)}{H_{\mathcal{F}}^{1}\left(\mathbb{Q}_{n, v}, A_{f, \eta}\right)}$ for each $v$. As mentioned after definition 3.2 we have $H_{\mathcal{F}}^{1}\left(\mathbb{Q}_{n, p}, A_{f, \eta}\left[p^{m}\right]\right)=H_{\mathcal{F}}^{1}\left(\mathbb{Q}_{n, p}, A_{f, \eta}\right)\left[p^{m}\right]$, thus $f_{p, m}$ is injective. Let $v$ be a prime not lying above $p$. From the Serre-Hochschild spectral sequence we can see that $\frac{H^{1}\left(\mathbb{Q}_{n, v}, A_{f, \eta}\left[p^{m}\right]\right)}{H_{\mathcal{F}}^{1}\left(\mathbb{Q}_{n, v}, A_{f, \eta}\left[p^{m}\right]\right)}$ and $\frac{H^{1}\left(\mathbb{Q}_{n, v}, A_{f, \eta}\right)}{H_{\mathcal{F}}^{1}\left(\mathbb{Q}_{n, v}, A_{f, \eta}\right)}$ are subgroups of $H^{1}\left(\mathbb{Q}_{n, v}^{u r}, A_{f, \eta}\left[p^{m}\right]\right)$ and $H^{1}\left(\mathbb{Q}_{n, v}^{u r}, A_{f, \eta}\right)$ respectively. From the long exact sequence induced from $A_{f, \eta}\left[p^{m}\right] \rightarrow A_{f, \eta} \stackrel{p^{m}}{\rightarrow} A_{f, \eta}$ we have

$$
A_{f, \eta}^{I_{v}} / p^{m} A_{f, \eta}^{I_{v}}=\operatorname{ker}\left(H^{1}\left(\mathbb{Q}_{n, v}^{u r}, A_{f, \eta}\left[p^{m}\right]\right) \rightarrow H^{1}\left(\mathbb{Q}_{n, v}^{u r}, A_{f, \eta}\right)\right) .
$$

Let $l$ be the residue characteristic of $v$ and fix an embedding $\overline{\mathbb{Q}} \rightarrow \mathbb{C}_{l}$ such that $v$ is the prime of $\mathbb{Q}_{n}$ corresponding to this embedding. Let $n^{\prime}$ be any integer bigger than $n$ and $w$ be the prime of $\mathbb{Q}_{n^{\prime}}$ corresponding to the embedding. Since $I_{v}=I_{w}$, we have $A_{f, \eta}^{I_{v}} / p^{m} A_{f, \eta}^{I_{v}}=A_{f, \eta}^{I_{w}} / p^{m} A_{f, \eta}^{I_{w}}$. In other words, $A_{f, \eta}^{I_{v}} / p^{m} A_{f, \eta}^{I_{v}}$ does not depend on $n$.

Furthermore, the size of $A_{f, \eta}^{I_{v}} / p^{m} A_{f, \eta}^{I_{v}}$ is bounded by the size of $A_{f, \eta}^{I_{v}} /\left(A_{f, \eta}^{I_{v}}\right)_{d i v}$. Since no prime splits completely over $\mathbb{Q}_{\infty} / \mathbb{Q}$, the kernel of $\prod_{v} f_{v, m}$ is bounded as $m, n$ vary. By the Snake Lemma our claim follows.

From lemmas 3.3 and 3.4 we have the following corollary.

Corollary 3.5. We have $\operatorname{corank}_{O} H_{\mathcal{F}}^{1}\left(\mathbb{Q}_{n}, A_{f, \eta}\right)=\operatorname{corank}_{O} H_{\mathcal{F}}^{1}\left(\mathbb{Q}_{n}, A_{f^{\iota}, \bar{\eta}}\right)$ for every n. Also $\left|H_{\mathcal{F}}^{1}\left(\mathbb{Q}_{n}, A_{\eta}\right)\left[p^{m}\right]\right| /\left|H_{\mathcal{F}}^{1}\left(\mathbb{Q}_{n}, A_{\bar{\eta}}\right)\left[p^{m}\right]\right|$ is bounded as $m, n$ vary.

We note the following proposition. 
Proposition 3.6 ([1] chapter 3$)$. Let $X$ and $Y$ be co-finitely generated $\Lambda_{O}$-modules. Assume that $X, Y$ satisfy

1. $\operatorname{corank}_{O}\left(X \otimes_{O} \Lambda_{O} /\left(f^{e}\right)\right)^{\Gamma}=\operatorname{corank}_{O}\left(Y \otimes_{O} \Lambda_{O} /\left(f^{e}\right)\right)^{\Gamma}$ for every monic irreducible distinguished polynomial $f(X) \in \Lambda_{O}$ and every $e<\infty$,

2. for every $e<\infty,\left|X^{\Gamma_{n}}\left[p^{e}\right]\right| /\left|Y^{\Gamma_{n}}\left[p^{e}\right]\right|$ is bounded as $n$ varies.

Then $X^{\vee}$ is pseudo-isomorphic to $Y^{\vee}$.

Using proposition 3.6 we prove the following.

Proposition 3.7. Let $X_{\eta}$ be $H_{\mathcal{F}}^{1}\left(\mathbb{Q}_{\infty}, A_{\eta}\right)^{\vee}$. Then we have $X_{\eta} \sim X_{\bar{\eta}}^{\iota}$ as $\Lambda_{O}$-modules.

Proof. Since $G_{\mathbb{Q}_{\infty}}$ acts trivially on $Y_{f}$ and $Y_{f}$ is a free $O$-module, we have

$$
\begin{aligned}
H_{\mathcal{F}}^{1}\left(\mathbb{Q}_{\infty}, A_{\eta}\right) \otimes Y_{f} & =\operatorname{ker}\left(H^{1}\left(\mathbb{Q}_{\Sigma} / \mathbb{Q}_{\infty}, A_{\eta}\right) \otimes Y_{f} \rightarrow \prod_{w} \frac{H^{1}\left(\mathbb{Q}_{\infty, w}, A_{\eta}\right) \otimes Y_{f}}{H_{\mathcal{F}}^{1}\left(\mathbb{Q}_{\infty, w}, A_{\eta}\right) \otimes Y_{f}}\right) \\
& =\operatorname{ker}\left(H^{1}\left(\mathbb{Q}_{\Sigma} / \mathbb{Q}_{\infty}, A_{f, \eta}\right) \rightarrow \prod_{w} \frac{H^{1}\left(\mathbb{Q}_{\infty, w}, A_{f, \eta}\right)}{H_{\mathcal{F}}^{1}\left(\mathbb{Q}_{\infty, w}, A_{\eta}\right) \otimes Y_{f}}\right)
\end{aligned}
$$

where $w$ runs over all the primes lying above $\Sigma$.

Using the Serre-Hochschild spectral sequence one can easily check

$$
H^{1}\left(\mathbb{Q}, A_{f, \eta}\right) \stackrel{\sim}{\rightarrow} H^{1}\left(\mathbb{Q}_{\infty}, A_{f, \eta}\right)^{\Gamma} .
$$

From the definition we have

$$
\left(H_{\mathcal{F}}^{1}\left(\mathbb{Q}_{\infty, p}, A_{\eta}\right) \otimes Y_{f}\right)^{\Gamma}=\left(\epsilon_{\bar{\eta}} \cdot \prod_{P \mid p} \mathbb{H}_{P} \otimes Y_{f}\right)^{\Gamma}=H_{\mathcal{F}}^{1}\left(\mathbb{Q}_{p}, A_{f, \eta}\right),
$$

thus we have an injection

$$
0 \rightarrow \frac{H^{1}\left(\mathbb{Q}_{p}, A_{f, \eta}\right)}{H_{\mathcal{F}}^{1}\left(\mathbb{Q}_{p}, A_{f, \eta}\right)} \rightarrow \frac{H^{1}\left(\mathbb{Q}_{\infty, p}, A_{f, \eta}\right)}{H_{\mathcal{F}}^{1}\left(\mathbb{Q}_{\infty, p}, A_{\eta}\right) \otimes Y_{f}} .
$$

For any prime $w$ of $\mathbb{Q}_{\infty}$ lying above a prime $v \neq p$ of $\mathbb{Q}$, we have $H^{1}\left(\mathbb{Q}_{v}^{u r}, A_{f, \eta}\right)=$ $H^{1}\left(\mathbb{Q}_{\infty, w}, A_{f, \eta}\right)$ because $\mathbb{Q}_{\infty, w} / \mathbb{Q}_{v}$ is a $\mathbb{Z}_{p}$-extension (in fact, the only $\mathbb{Z}_{p}$-extension and the only unramified $\mathbb{Z}_{p}$-extension). For the same reason we have

$$
H^{1}\left(\mathbb{Q}_{\infty, w}^{u r} / \mathbb{Q}_{\infty, w}, A_{\eta}^{G_{\mathbb{Q}}^{\text {ur }}, w}\right)=H_{\mathcal{F}}^{1}\left(\mathbb{Q}_{\infty, w}, A_{\eta}\right)=0
$$

Thus we have

$$
\frac{H^{1}\left(\mathbb{Q}_{\infty, w}, A_{\eta}\right) \otimes Y_{f}}{H_{\mathcal{F}}^{1}\left(\mathbb{Q}_{\infty, w}, A_{\eta}\right) \otimes Y_{f}}=\frac{H^{1}\left(\mathbb{Q}_{\infty, w}, A_{\eta}\right) \otimes Y_{f}}{H_{u r}^{1}\left(\mathbb{Q}_{\infty, w}, A_{\eta}\right) \otimes Y_{f}}=H^{1}\left(\mathbb{Q}_{\infty, w}, A_{f, \eta}\right)=H^{1}\left(\mathbb{Q}_{v}^{u r}, A_{f, \eta}\right) .
$$

Since $\frac{H^{1}\left(\mathbb{Q}_{v}, A_{f, \eta}\right)}{H_{\mathcal{F}}^{1}\left(\mathbb{Q}_{v}, A_{f, \eta}\right)} \rightarrow H^{1}\left(\mathbb{Q}_{v}^{u r}, A_{f, \eta}\right)$ is an injection by the definition of $H_{\mathcal{F}}^{1}$, we have an injection

$$
0 \rightarrow \frac{H^{1}\left(\mathbb{Q}_{v}, A_{f, \eta}\right)}{H_{\mathcal{F}}^{1}\left(\mathbb{Q}_{v}, A_{f, \eta}\right)} \rightarrow \frac{H^{1}\left(\mathbb{Q}_{\infty, w}, A_{\eta}\right) \otimes Y_{f}}{H_{\mathcal{F}}^{1}\left(\mathbb{Q}_{\infty, w}, A_{\eta}\right) \otimes Y_{f}}
$$


From $(2),(3),(4)$, and the snake lemma we can see $H_{\mathcal{F}}^{1}\left(\mathbb{Q}, A_{f, \eta}\right)=\left(H_{\mathcal{F}}^{1}\left(\mathbb{Q}_{\infty}, A_{\eta}\right) \otimes\right.$ $\left.Y_{f}\right)^{\Gamma}$.

Combined with corollary 3.5 we have

$$
\operatorname{corank}_{O}\left(H_{\mathcal{F}}^{1}\left(\mathbb{Q}_{\infty}, A_{\eta}\right) \otimes Y_{f}\right)^{\Gamma}=\operatorname{corank}_{O}\left(H_{\mathcal{F}}^{1}\left(\mathbb{Q}_{\infty}, A_{\bar{\eta}}\right) \otimes Y_{f^{\iota}}\right)^{\Gamma} .
$$

Similarly we can check $H_{\mathcal{F}}^{1}\left(\mathbb{Q}_{n}, A_{\eta}\right)=H_{\mathcal{F}}^{1}\left(\mathbb{Q}_{\infty}, A_{\eta}\right)^{\Gamma_{n}}$. By corollary 3.5 we can see $\left|H_{\mathcal{F}}^{1}\left(\mathbb{Q}_{\infty}, A_{\eta}\right)^{\Gamma_{n}}\left[p^{m}\right]\right| /\left|H_{\mathcal{F}}^{1}\left(\mathbb{Q}_{\infty}, A_{\bar{\eta}}\right)^{\Gamma_{n}}\left[p^{m}\right]\right|$ is bounded as $m$ and $n$ vary.

By proposition 3.6 our claim follows.

We let $H_{\mathcal{F}}^{1}\left(K_{\infty, P}, A\right):=\mathbb{H}_{P} \otimes O$ and $H_{\mathcal{F}}^{1}\left(K_{\infty, P}, E\left[p^{\infty}\right]\right):=\mathbb{H}_{P}$ for $P \mid p$, and let $H_{\mathcal{F}}^{1}\left(K_{\infty, w}, A\right):=H_{u r}^{1}\left(K_{\infty, w}, A\right)$ and $H_{\mathcal{F}}^{1}\left(K_{\infty, w}, E\left[p^{\infty}\right]\right):=H_{u r}^{1}\left(K_{\infty, w}, E\left[p^{\infty}\right]\right)$ for primes $w$ not lying above $p$. We define a group

$$
S_{p}^{-}\left(A / K_{\infty}\right):=\operatorname{ker}\left(H^{1}\left(K_{\Sigma} / K_{\infty}, A\right) \rightarrow \prod_{w} \frac{H^{1}\left(K_{\infty, w}, A\right)}{H_{\mathcal{F}}^{1}\left(K_{\infty, w}, A\right)}\right),
$$

and the minus Selmer group

$$
\operatorname{Sel}_{p}^{-}\left(E / K_{\infty}\right):=\operatorname{ker}\left(H^{1}\left(K_{\Sigma} / K_{\infty}, E\left[p^{\infty}\right]\right) \rightarrow \prod_{w} \frac{H^{1}\left(K_{\infty, w}, E\left[p^{\infty}\right]\right)}{H_{\mathcal{F}}^{1}\left(K_{\infty, w}, E\left[p^{\infty}\right]\right)}\right)
$$

where $w$ runs over all the places lying above $\Sigma$. We can easily check $S_{p}^{-}\left(A / K_{\infty}\right)=$ $\operatorname{Sel}_{p}^{-}\left(E / K_{\infty}\right) \otimes O$ (compare this definition with that of [5], [2], and [4]).

We note that the definitions of $H_{\mathcal{F}}^{1}\left(\mathbb{Q}_{\infty}, A_{\eta}\right)$ and $S_{p}^{-}\left(A / K_{\infty}\right)$ do not depend on the choice of $\Sigma$. Indeed when we take all places of $\mathbb{Q}$ for $\Sigma$, we still have the same $H_{\mathcal{F}}^{1}\left(\mathbb{Q}_{\infty}, A_{\eta}\right)$ and $S_{p}^{-}\left(A / K_{\infty}\right)$. We consider the following diagram:

$$
\begin{aligned}
& 0 \rightarrow H_{\mathcal{F}}^{1}\left(\mathbb{Q}_{\infty}, A_{\eta}\right) \rightarrow H^{1}\left(\mathbb{Q}_{\infty}, A_{\eta}\right) \rightarrow \prod_{v} \frac{H^{1}\left(\mathbb{Q}_{\infty, v}, A_{\eta}\right)}{H_{\mathcal{F}}^{1}\left(\mathbb{Q}_{\infty, v}, A_{\eta}\right)} \\
& \begin{array}{ccc}
\downarrow & \downarrow & \downarrow \prod_{1} f_{v} \\
0 \rightarrow \quad S_{p}^{-}\left(A / K_{\infty}\right)^{\bar{\eta}} \rightarrow & H^{1}\left(K_{\infty}, A\right)^{\bar{\eta}} \rightarrow & \prod_{w} \frac{H^{1}\left(K_{\infty, w}, A\right)}{H_{\mathcal{F}}^{1}\left(K_{\infty, w}, A\right)}
\end{array}
\end{aligned}
$$

where $v$ and $w$ run over all places of $\mathbb{Q}_{\infty}$ and $K_{\infty}$ respectively.

Since $A^{G_{K_{\infty}, P}}=0$ for any prime $P$ lying above $p$, we have $A^{G_{K_{\infty}}}=0$. Using that, we have $H^{1}\left(K_{\infty}, A\right)^{\bar{\eta}}=H^{1}\left(K_{\infty}, A_{\eta}\right)^{\operatorname{Gal}(K / \mathbb{Q})}=H^{1}\left(\mathbb{Q}_{\infty}, A_{\eta}\right)$ by the SerreHochschild spectral sequence. From the definition of $H_{\mathcal{F}}^{1}\left(\mathbb{Q}_{\infty, p}, A_{\eta}\right)$ we can see $f_{p}$ is an injection. Recall that when $v$ and $w$ are not lying above $p$, we have $H_{\mathcal{F}}^{1}\left(\mathbb{Q}_{\infty, v}, A_{\eta}\right)=$ $H_{\mathcal{F}}^{1}\left(K_{\infty, w}, A\right)=0$. Since the order of $\operatorname{Gal}\left(K_{\infty, w} / \mathbb{Q}_{\infty, v}\right)$ is prime to $p$, we have

$$
\operatorname{ker}\left(H^{1}\left(\mathbb{Q}_{\infty, v}, A_{\eta}\right) \rightarrow H^{1}\left(K_{\infty, w}, A_{\eta}\right)\right)=H^{1}\left(K_{\infty, w} / \mathbb{Q}_{\infty, v}, A_{\eta}^{G_{K_{\infty}, w}}\right)=0 .
$$

Thus we have the following.

Proposition 3.8. We have $H_{\mathcal{F}}^{1}\left(\mathbb{Q}_{\infty}, A_{\eta}\right) \cong S_{p}^{-}\left(A / K_{\infty}\right)^{\bar{\eta}}$.

The next proposition is a simple consequence of Rohrlich and Kato's work. 
Proposition 3.9. $\operatorname{Sel}_{p}^{-}\left(E / K_{\infty}\right)$ is $\Lambda$-cotorsion.

Proof. For a finite group $G$, a character $\chi$ of $G$, and a $\mathbb{Z}_{p}[G]$-module $M$ we let $M^{\chi}$ be the $\chi$-part of $M \otimes \mathbb{Z}_{p}[\chi]$. By [4] lemma 4.20 there is an integer $N$ such that for any $n>N$ with odd $n-N$ and a primitive character $\chi$ of $\operatorname{Gal}\left(K_{n} / K_{N}\right)$ we have

$$
\operatorname{corank}_{\mathbb{Z}_{p}[\chi]} \operatorname{Sel}_{p}\left(E / K_{n}\right)^{\chi}=\operatorname{rank}_{\mathbb{Z}_{p}[\chi]}\left(\operatorname{Sel}_{p}^{-}\left(E / K_{\infty}\right)^{\Gamma_{n}}\right)^{\chi} .
$$

By Rohrlich $([8],[9]) L(E / \mathbb{Q}, \chi, 1) \neq 0$ for all but finitely many Dirichlet characters $\chi$ of $\operatorname{Gal}\left(K_{n} / \mathbb{Q}\right)$ as $n$ varies. By Kato $([3])$, if $L(E / \mathbb{Q}, \chi, 1) \neq 0$, we have $\operatorname{corank}_{\mathbb{Z}_{p}[\chi]} \operatorname{Sel}_{p}\left(E / K_{n}\right)^{\chi}=0$. Therefore there are infinitely many integers $n$ such that $\operatorname{corank}_{\mathbb{Z}_{p}[\chi]}\left(\operatorname{Sel}_{p}^{-}\left(E / K_{\infty}\right)^{\Gamma_{n}}\right)^{\chi}=0$ for any primitive character $\chi$ of $\operatorname{Gal}\left(K_{n} / K_{N}\right)$. Thus $\operatorname{Sel}_{p}^{-}\left(E / K_{\infty}\right)$ is $\Lambda$-cotorsion.

Combined with propositions 3.7 and 3.8 we have the following.

Theorem 3.10. Let $X=\left(\operatorname{Sel}_{p}^{-}\left(E / K_{\infty}\right) \otimes O\right)^{\vee}$. For each character $\eta$ of $\Delta$ we have $X^{\eta} \sim X^{\iota, \bar{\eta}}$ as $\Lambda_{O}$-modules, or equivalently $X \sim X^{\iota}$ as $O\left[\left[\mathrm{Gal}\left(K_{\infty} / \mathbb{Q}\right)\right]\right]$-modules. Consequently we have the following: let $(a) \subset \Lambda$ be the characteristic ideal of $\operatorname{Sel}_{p}^{-}\left(E / K_{\infty}\right)^{\vee}$. We have $(a)=\left(a^{\iota}\right)$.

It is more tricky to deal with $\operatorname{Sel}_{p}^{+}\left(E / K_{\infty}\right)$. Although it is not explained in [4], it is not clear that the plus norm subgroup in [4] always has the property the minus norm subgroup has. More specifically (using the notation of [4] propositions 3.12 and 3.13) it is not clear that the set $\left\{c_{0, i}\right\}_{0,1, \cdots, d-1}$ linearly generates $\hat{E}(m)$. Consequently it is not clear that we have $\left(\cup_{n=1}^{\infty} \hat{E}^{+}\left(m_{k_{n}}\right) \otimes \mathbb{Q}_{p} / \mathbb{Z}_{p}\right)^{\vee} \cong \Lambda^{d}$. However, Kobayashi's plus/minus norm subgroups for $\mathbb{Q}\left(\mu_{p^{\infty}}\right)$ have this property. We can apply our technique to both \pm -Selmer groups with little difficulty to obtain the following.

Theorem 3.11. We have

$$
\operatorname{Sel}_{p}^{ \pm}\left(E / \mathbb{Q}\left(\mu_{p^{\infty}}\right)\right)^{\vee} \sim S e l_{p}^{ \pm}\left(E / \mathbb{Q}\left(\mu_{p^{\infty}}\right)\right)^{\vee, \iota}
$$

where $\sim$ is a pseudoisomorphism for $\mathbb{Z}_{p}\left[\left[\operatorname{Gal}\left(\mathbb{Q}\left(\mu_{p^{\infty}}\right) / \mathbb{Q}\right)\right]\right]$-modules.

Iovita and Pollack's plus/minus norm subgroups also work well under the following condition: The prime $p$ splits completely over $K / \mathbb{Q}$. Note that any prime of $K$ lying above $p$ is totally ramified in the cyclotomic $\mathbb{Z}_{p}$-extension $K_{\infty}$. Assuming this condition we can prove a similar result.

Theorem 3.12. We have

$$
\left(S e l_{p}^{ \pm}\left(E / K_{\infty}\right) \otimes O\right)^{\vee} \sim\left(S e l_{p}^{ \pm}\left(E / K_{\infty}\right)^{\iota} \otimes O\right)^{\vee}
$$

\section{Acknowledgements}

The author is grateful to Karl Rubin, Ralph Greenberg, and Robert Pollack for many insightful conversations. He is also grateful to the anonymous referee for many good suggestions. 


\section{References}

[1] R. Greenberg, Iwasawa theory for p-adic representations, Adv. Stud. Pure Math. (1989), no. 17, 97-137.

[2] A. Iovita and R. Pollack, Iwasawa theory of elliptic curves at supersingular primes over $\mathbb{Z}_{p}$ extensions of number fields, to appear in Crelle.

[3] K. Kato, p-adic Hodge theory and values of zeta functions of modular forms (2004), no. 295, 117-290.

[4] B. Kim, The parity conjecture for elliptic curves at supersingular reduction primes, Compositio Math. (2007), no. 143, 47-72.

[5] S. Kobayashi, Iwasawa theory for elliptic curves at supersingular primes, Invent. Math. (2003, no. 1), no. $152,1-36$.

[6] J. Milne, Arithmetic duality theorems, Perspectives in Math, 1 (1986)

[7] R. Pollack, On the p-adic L-function of a modular form at a supersingular prime, Duke Math. Journal (2003 no. 3), no. 118, 523-558.

[8] D. Rohrlich, On L-functions of elliptic curves and cyclotomic towers, Invent. Math. (1984), no. $75,404-423$.

[9] —, L-functions and division towers, Math. Ann. (1988), no. 281, 611-632.

[10] J. Tate, Duality theorems in Galois cohomology over number fields, Proc. Intern. Cong. Math. (1962)

Department of Mathematics, Northwestern University, 2033 Sheridan Road, Evanston, IL, 60208 U.S.A.

E-mail address: bdkim@math.northwestern.edu 